\title{
Design and Simulation Analysis of Multi-Agent Online Dissemination Model on the Basis of "The Spiral of Silence" Theory
}

\author{
Qiuyun Miao ${ }^{1} \&$ Yang Shen ${ }^{1}$ \\ ${ }^{1}$ School of Economics and Management, Nanjing University of Aeronautics and Astronautic, China \\ Correspondence: Yang Shen, School of Economics and Management, Nanjing University of Aeronautics and \\ Astronautic, Nanjing, China. Tel: 1-381-390-5870. E-mail: shen.y@nuaa.edu.cn
}

Received: September 30, 2015

Accepted: October 16, $2015 \quad$ Online Published: October 31, 2016

doi:10.5539/cis.v9n4p44

URL: http://dx.doi.org/10.5539/cis.v9n4p44

\begin{abstract}
This article studies the classical theory of communications - the Spiral of Silence. The key factors are extracted for multi-agent modeling and simulation with Netlogo. It turns out that opinion climate is the external formation of the Spiral of Silence. Besides, the size of opinion climate has no direct effects on the Spiral of Silence.
\end{abstract}

Keywords: the Spiral of Silence, multi-agent modeling, simulation, Netlogo

\section{Introduction}

The innovation of "The Spiral of Silence" theory has attracted many researchers under today's network communication environment, some scholars believe that the the Spiral of Silence in cyberspace is weakening which plays a new role in network media. GAO Xianchun et al. think that: "Mass media and specific individual communities make a difference in the new media environment constructed by Internet, mobile phones and others which are in the construction of specific event. And they both trigger the double helix of silence. In other words, mass media and individual communities form spirals respectively, meanwhile, both games lead to opinion cassette which is the factor affecting individual perception. As a result, the double helix of silence influences individual expressions of opinion. "(GAO Xianchun et al,2014) Yang Zhibiao supports the thory despite of its assumptions and limitations. In his opinion, it is still available in network environment. What is more, "the spiral of silence" is alive with "Against the Spiral of Silence".

However, other scholars believe that the Spiral of Silence is still spinning in the new era. To explore the views of "Gay Bullying", Sherice Gearhart et al. investigate 760 participants. It was found that the the Spiral of Silence still exists in social networks, besides, personal ideology will also have an impact on the willingness of expression (Sherice Gearhart et al, pp. 18-36, 2014. ). Li Chuansheng points out that the Spiral of Silence still works because of the fear of isolation and group pressure in the network. What is more, the Internet has a character of anonymity, but net users' behavior is not unrestricted and complete freedom does not exist which due to group norm and social norm in the network community. That is the reason why the Spiral of Silence is still in the spin (Li Chuansheng, pp. 4-12, 2014). Na Yeon Lee et al. carry out a series of studies on controversial topics among journalists who work for nine national newspapers and two networks broadcasting corporation through the empirical research. It turns out that the Spiral of Silence still exists, especially in the social networking site-Twitter (YL Na, pp. 443-458, 2015). Cheng Yao also points out that because of unconverted habits of netizens, squelch on the Internet and attention which the country begins paying to supervising public opinion online, the Spiral of Silence will coexist with public opinion on the network for a long time in a more subtle way. Moreover, according to a report released by the Pew Research Center and the Rutgers University, social media such as Twitter and Facebook will contain the diversification of views, hinder public affairs debates and limit people to state their views. Especially when they find that their opinions are different the fear of isolation; climate of opinion, and quasi-statistical sensewith friends'. The report also said that very few people who use social media regularly express different points of view offline.

In order to study the impacts mechanisms of the Spiral of Silence, Scholars adopt empirical research through questionnaire to explore the impact of " the climate of opinion " - one factor of the Spiral of Silence. In Chang Ning's research, it is found that post-90 college students adhere to the "expression"of the highest degree in real life. Unexpectedly, under the anonymous network, post-90 college students tend be more silence. The study also shows that the anonymity of the network is not the main factor to promote the expression. Zhou Baohua 
investigates the willingness of college students and its factors by sampling survey. Based on the test results and analysis, different climate of opinion has different effects on college students which is suggested. The degree of consensus with the majority in society and those online has no obvious effects on the expression of college students on the Internet. However, opinions of college students are not the same as those of main medium or those of parents', the network has a significant positive effect on college students to express their opinions.

Above all, the Spiral of Silence may not be fully consistent with the characteristics of dissemination of public opinion in the new era, but it still works. At the same time, there are few papers exploring the propagation mechanism of the Spiral of Silence by multi-agent modeling. The paper attempts to describe communicating characteristics of the Spiral of Silence from relatively objective perspectives. At first, this paper will construct propagation model of the Spiral of Silence by using multi-agent modeling. Then, the paper adopts Netlogo to simulate the model. Finally, the model will be validated.

\section{Framework of the Digest Format}

The Spiral of Silence theory put forward by German communication scholar Elisabeth Noelle-Neumann explains how people decide whether or not to express their own true feelings. According to the theory, the will of expression is associated with external pressure, such as the media and the views of people around. The spiral of silence is widely used in media and communication research to describe the silence of controversial events.

In 1980, Elisabeth Noelle-Neumann published The Spiral of Silence: Public Opinion-Our Social Skin, she thought the spiral of silence comes from the nature of the society, at the same time, it comes from entrenched fear of human beings. When people found himself/herself is a member of the minority, he/she would tend to be silence because of fear of isolation. On the contrary, when a person is a member of the majority, he/she would be more willing to express himself/herself. In this book, she thought that the theory was made up of five basic hypotheses in summary.

(1)Individual fear of isolation;

(2)The individual evaluates the climate of opinion constantly because of fear of isolation.

(3)The fear of isolation is used to deal with individuals who deviate from the mainstream by the society.

(4)The results of evaluation affect individual behavior and opinion in public, especially by revealing or hiding their views.

(5)This hypothesis relates to four hypotheses above and they combine to explain the formation and change of public opinion and so on. (Noelle-Neumann, pp. 143-158, 1977)

Due to the fear of isolation, the agents will change their opinions according to majority opinion. To avoid isolation, people will try to seek the mainstream views and observe the rally of all kinds of views. Repeat the cycle, one voice is growing, while the other tends to be silence which is called the Spiral of Silence.

\section{Modeling and Simulation based on the Theory}

\subsection{Modeling based on the Spiral of Silence}

According to Neumann's theory, there are three main factors of the theory: the fear of isolation; climate of opinion and quasi-statistical sense. The paper summarizes model as shown in Figure1.

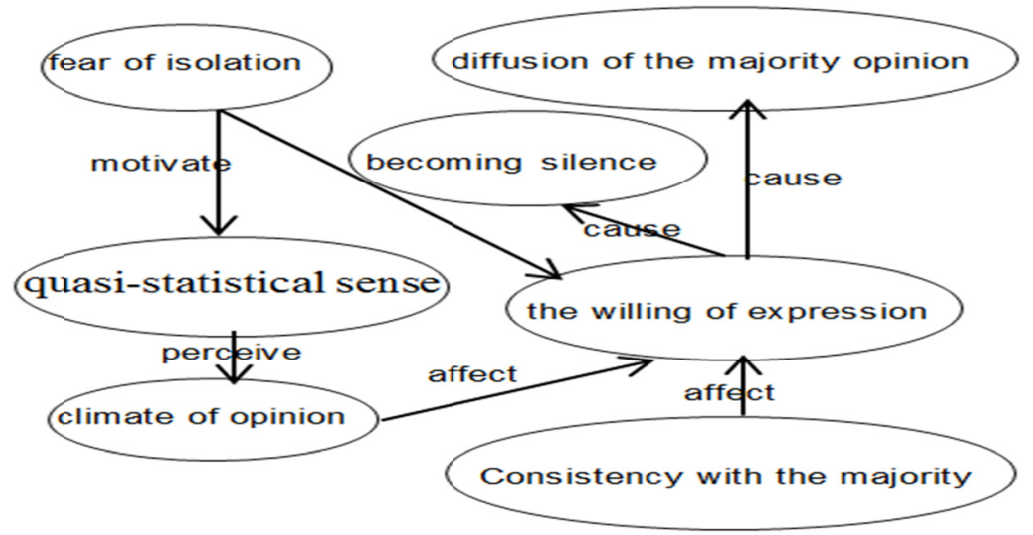

Figure 1. Model of the Spiral of Silence. 
According to the model, the ultimate source of the Spiral of Silence is fear of isolation, but "climate of opinion" and "consistency with the majority" can affect the willing of expression directly. In order to facilitate research, this paper raises some brief and necessary assumptions:

Hypothesis 1: There are only two sides in the dissemination of public opinion. That is to say there are only positive opinion and negative opinion. At the same time, climate of opinion can be simulated by adjusting the ratio of positive and negative comments.

Hypothesis 2: There is only one kind of the majority opinion online. On the basis of the theory, there is always an overwhelming view in the dissemination of public opinion. Thus, adjusting the proportion of two sides of the opinion by which the majority opinion can be randomly decided in the Internet can simulate the propagation mechanism of the Spiral of Silence well.

Hypothesis 3: In given conditions, Individual views may change along with the spread of public opinion.

There are two main agents in the construction of simulation model, as shown in Figure 2.

\begin{tabular}{|l|l|}
\hline \multicolumn{1}{|c|}{ turtles } \\
\hline $\begin{array}{l}\text { afraid-index } \\
\text { interest }\end{array}$ \\
\hline $\begin{array}{l}\text { change-afraid-index } \\
\text { change-color } \\
\text { get-uninterested }\end{array}$ \\
\hline
\end{tabular}

Figure 2. Class Diagram

The agent "turtle" is made up of two attributes: afraid-index and interest. When external climate of opinion changes, individual afraid-index will change accordingly and agents may change their opinions. Setting methods includes the followings: "change-afraid-index"、"change-color" (Color changes indicate the changing of opinion in the program) and "get-interest". Properties of the agent "patch" consist of "green-turtles"、"red-turtles" and "radius". Properties are used to count the number of green and red turtles within a radius of "radius" around a certain "patch" in order to simulate the climate of opinion around a certain "turtle" and the method "count-around-num" is needed to achieve the goal.

\subsection{Simulation based on the Spiral of Silence}

\section{(1) Properties Settings}

The fear of isolation and external climate of opinion affect the circumstances of information dissemination system in the theory which prompts the views of some events, evaluations and opinions in society. The evolution of Spiral of silence online exists in complex social network. The "Agent" can be seen as main element of view changes in this network. Attitude changes of netizens can be reflected by the change of the "Agent". The "Agent" influences each other through interactions which causes individual to change opinions. The study designs agent's properties in Table I:

Table 1. Introduction of Property Settings

\begin{tabular}{|c|c|c|}
\hline Agent & variable name & explanation \\
\hline \multirow[t]{5}{*}{ Turtles } & Initial-people & value scope $[0,1000]$ \\
\hline & & representing the number of people in the initial state \\
\hline & Initial-chance & value scope $[0,1]$ \\
\hline & & representing the probability distribution of initial state \\
\hline & $\begin{array}{l}\text { r-afraid-index } \\
\text { g-afraid-index }\end{array}$ & $\begin{array}{l}\text { representing red or green turtles' fear index and it will change as the } \\
\text { climate changes. }\end{array}$ \\
\hline \multirow[t]{3}{*}{ Patches } & red-turtles & representing the number of green and red turtles within a radius of \\
\hline & radius & value scope $[1,10]$ \\
\hline & & $\begin{array}{l}\text { representing the radius of the climate of opinion in which the turtle } \\
\text { can be affected }\end{array}$ \\
\hline
\end{tabular}


Most Internet users will try to avoid isolation caused by separate attitudes and views. When agents find their own views are different from those of most Internet users or those of the mainstream media, they will not tend to express their opinions. Instead, they may choose to change views to correspond to the mainstream views online.

(2) Settings of initial state

The slider--initial-chance is set to represent the distribution of initial state. The proportion of positive and negative opinions will vary when setting different ratios. Besides, The "initial-people" stands for the number of people who know the event and express their views. The "radius" is on behalf of the size of the climate of opinion in which the agent can be affected. According to the spiral of silence, the fear of isolation is inherent nature of the people, thus the initial values of "r-afraid-index" and "g-afraid-index" are set to 50 each.

(3) Settings of interaction rules

a. Collecting Statistics of climate of opinion around some individual: through Netlogo program, the climate of opinion can be collected automatically. (Here's the code.)

to count-around-num

ask turtles [set red-turtles(sum[count turtles with [color $=$ red] ]of turtles in-radius radius) end

set green-turtles( sum[count turtles with [color $=$ green] ]of turtles in-radius radius)]

b. Changing Attitudes: when the value of the fear of isolation reaches a certain critical value, Internet users may change their views. What is more, when corresponding to the majority, agents will be willing to express their opinions and try to influence other agents. That's why there is a process called "hatch", here's the code.

to change-color if red-turtles $>$ green-turtles [

if color $=$ green and random $r$-afraid-index $<35$

[set color red fd random 10

hatch 1 [set color red fd random 10 set r-afraid-index 50 ]]

end

c. The ultimate source--"fear of isolation": the fear index will change accordingly when the individual is affected by the surroundings. In addition, different agents have different degrees of changes on the fear index. This part of the code is as follows:

to change-afraid-index

if red-turtles $>$ green-turtles

[set r-afraid-index(r-afraid-index - random 10)

set g-afraid-index(g-afraid-index + random 10)]

if red-turtles $<$ green-turtles

[set g-afraid-index(g-afraid-index - random 10)

set r-afraid-index(r-afraid-index + random 10)]

end

3.3 Analysis of Experimental Results

The initial value of " initial-people" is set to 880 which represents the number of people knowing the event at the first time. The initial value of " radius" is set to 1 . The percentage of two sides is randomly decided and different percentages have direct impacts on future climate trends. In this paper, the values of initial-chance are $0.1,0.3$, $0.5,0.7$ and 0.9 respectively. Parameters are shown in Table II..

Table 2. Parameter 1

\begin{tabular}{llllll}
\hline No. & initial-people & initial-chance & radius & r-afraid-index & g-afraid-index \\
\hline 1 & 880 & 0.1 & 1 & 50 & 50 \\
2 & 880 & 0.3 & 1 & 50 & 50 \\
3 & 880 & 0.5 & 1 & 50 & 50 \\
4 & 880 & 0.7 & 1 & 50 & 50 \\
5 & 880 & 0.9 & 1 & 50 & 50 \\
\hline
\end{tabular}




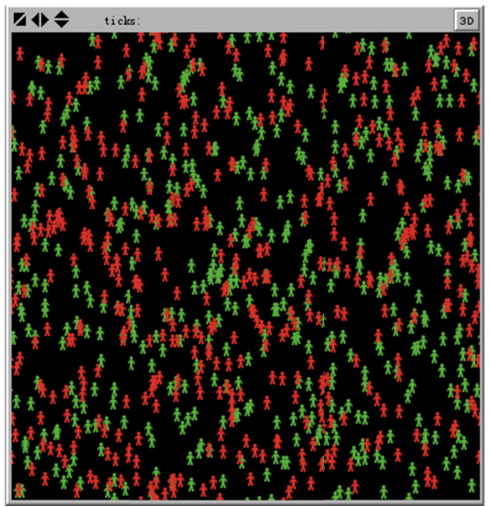

Experiment 1

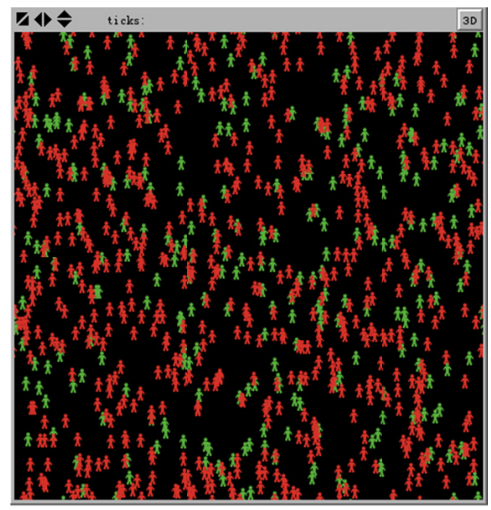

Experiment 2

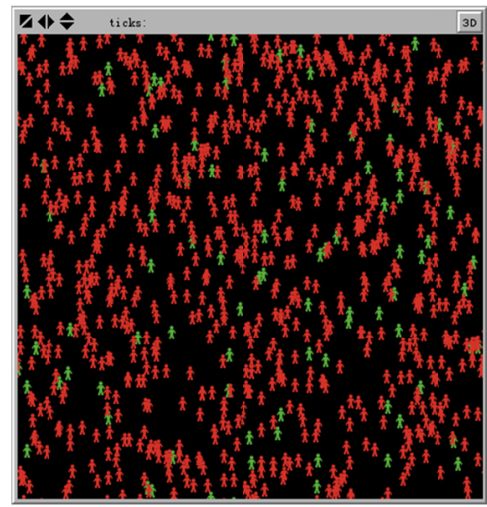

Experiment 3

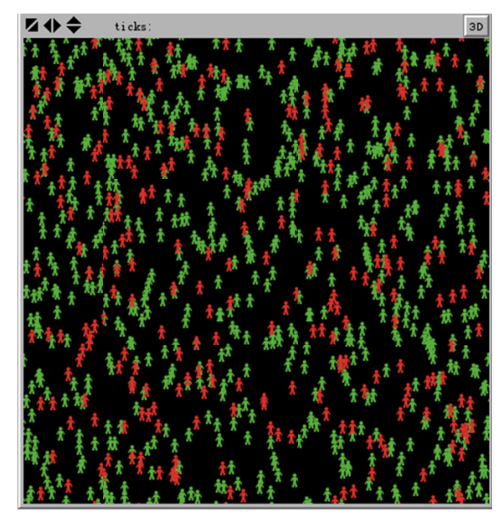

Experiment 3

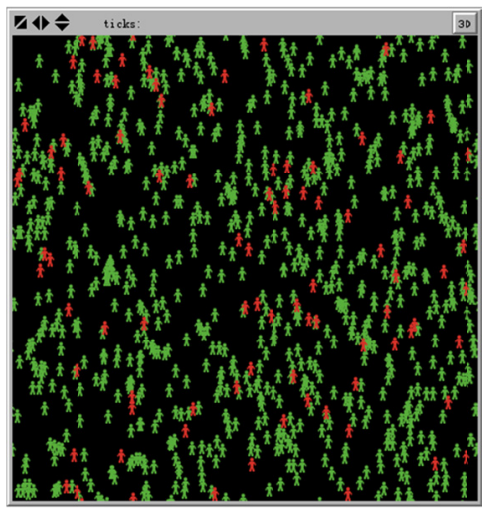

Experiment 4

Figure 3. initial interfaces corresponding to different proportions

Then, Press the "go" button to start running the program. Agents with different views in the interface will begin to change, and the corresponding graphs also began to be painted to show the change of different opinions as follows.

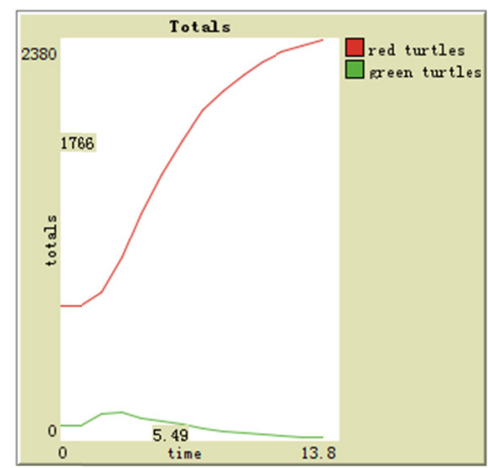

Experiment 1

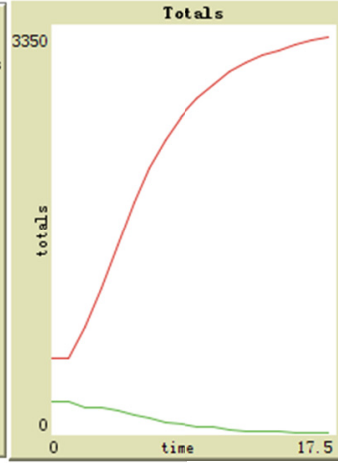

Experiment 2

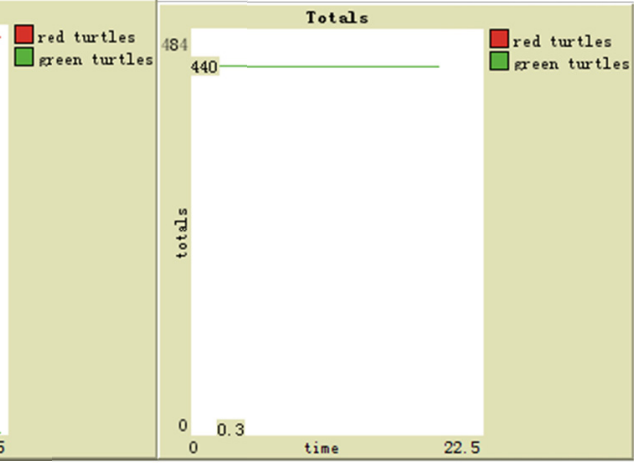

Experiment 3 


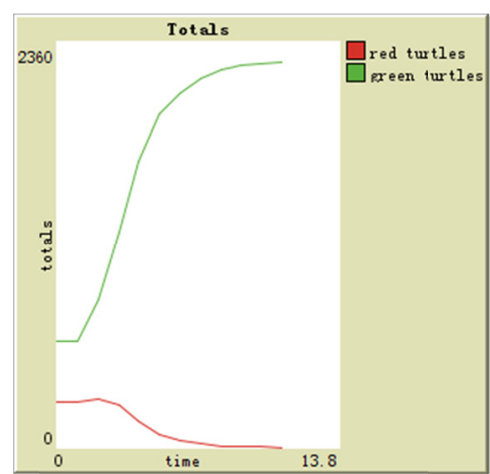

Experiment 4

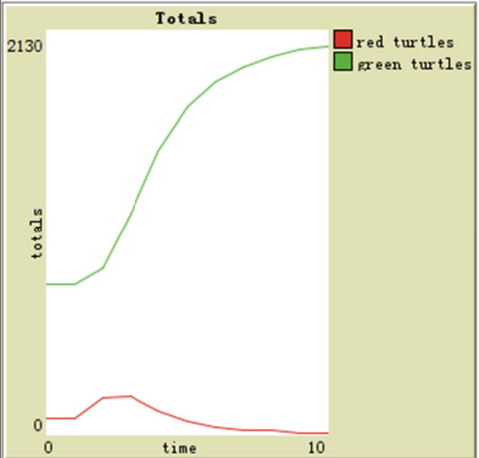

Experiment 5

Figure 4. change curve chart

As can be seen in Figure 4., characteristics of spiral of silence are reflected clearly regardless of different opinion ratios in the initial state $(0.1,0.3,0.7$ or 0.9$)$ : The "majority" opinion keeps increasing, while the "minority" opinion represents significantly fluctuate declining. When the value of "initial-chance" is 0.5 , the spiral of silence does not work. It can be concluded that uneven distribution of opinions is necessary for the theory, and that is to say, the distribution of views affects the formation of spiral of silence. Besides, it can be seen from the picture above that the climate of opinion is able to make the majority opinion strengthen, while it can make the minority opinion weaken.

In order to study the relationship between the theory and the parameter-radius which stands for the radius of the climate of opinion in which the turtle can be affected, five groups of experimental parameters are set up for the study, as shown below.

Table 3. Parameter 2

\begin{tabular}{llllll}
\hline No. & initial-people & initial-chance & radius & r-afraid-index & g-afraid-index \\
\hline $\mathbf{1}$ & 880 & 0.1 & 1 & 50 & 50 \\
$\mathbf{2}$ & 880 & 0.1 & 4 & 50 & 50 \\
$\mathbf{3}$ & 880 & 0.1 & 7 & 50 & 50 \\
$\mathbf{4}$ & 880 & 0.1 & 10 & 50 & 50 \\
\hline
\end{tabular}

Through these experiments, corresponding changes of curves are shown as below:

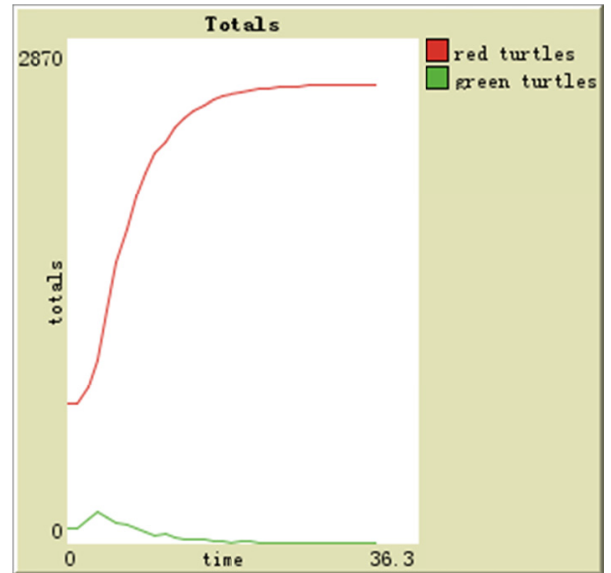

Experiment 1

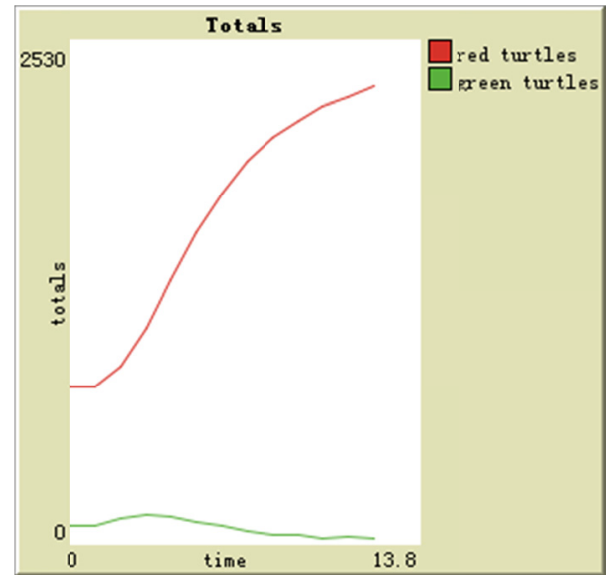

Experiment 2 


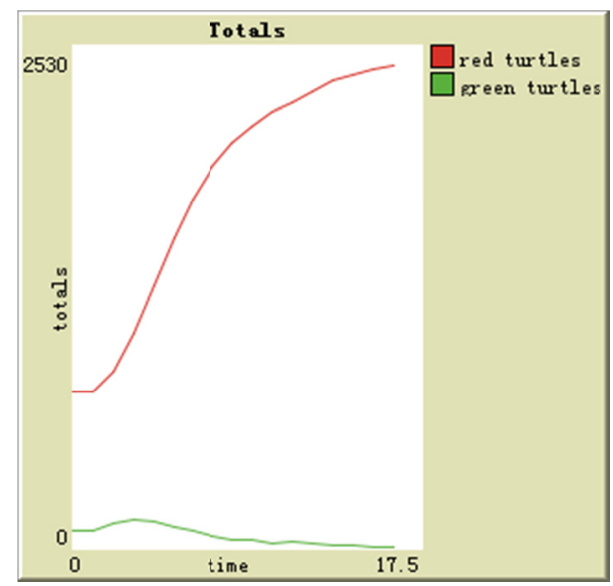

Experiment 3

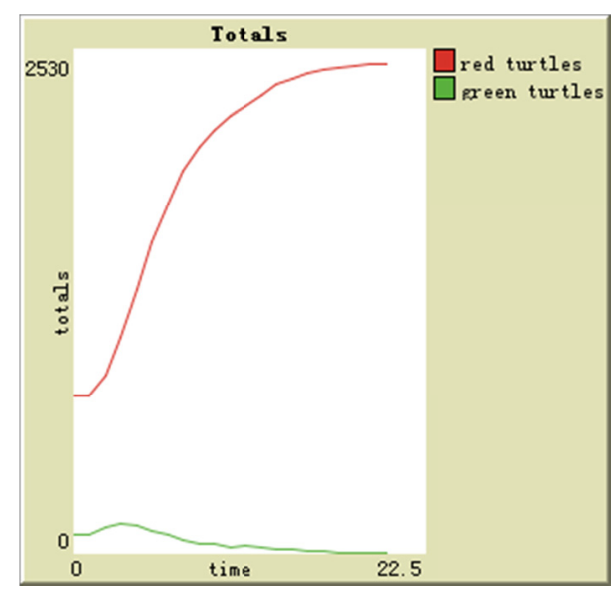

Experiment 4

Figure 5. change curves corresponding to different radius

It is easy to find that the size of climate of opinion does not affect the formation of spiral of silence. To explore the inner mechanism and the relationship between the size of climate of opinion and the rate of opinion changes, a few comparative tests were conducted. Apart from the value of "radius" other parameters are invariant. The results are summarized in Table IV :

Table 4. Research between radius and opinions change rate

\begin{tabular}{lllllll}
\hline radius & $\mathrm{R}^{(1)}$ & $\mathrm{R}^{(2)}$ & $\mathrm{G}^{(1)}$ & $\mathrm{G}^{(1)}$ & $\mathrm{R}^{(\mathrm{AVG})}$ & $\mathrm{G}^{(\mathrm{AVG})}$ \\
\hline 1 & 792 & 2014 & 88 & 69 & 174.57 & 2.71 \\
4 & 792 & 1998 & 88 & 70 & 172.28 & 2.57 \\
7 & 792 & 1975 & 88 & 75 & 169 & 1.86 \\
10 & 792 & 1987 & 88 & 71 & 170.71 & 2.42 \\
\hline
\end{tabular}

$\mathrm{R}^{(1)}$ represents the number of red-turtles at first; $\mathrm{R}^{(2)}$ represents the number of red-turtles after 7 seconds; $\mathrm{G}^{(1)}$ represents the number of green-turtles at first; $G^{(2)}$ represents the number of green-turtles after 7 seconds; $R^{(A V G)}$ represents red-turtles' average rate of rise; $G^{(A V G)}$ represents green-turtles' average rate of rise.

There is no direct link between the radius and the change rate of opinion. Thus, it can be concluded that the size of climate of opinion has no direct impact on the formation of spiral of silence.

\section{Conclusion}

The paper is based on the framework of the spiral of silence. At the same time, the study simulates the theory and analyses it. We can draw the following conclusions experiments: (1) The spiral of silence can make the majority opinion grow in strength while the minority opinion tend to die out finally; (2) The climate of opinion is the external factor of the spiral of silence; (3) The size of climate of opinion has no direct effects on the formation of spiral of silence.

\section{References}

Chang, N. (2015). Complex Performance of the Spiral of silence theory in the new media environment", Journal of Communication University of China, 6, 109-113.

Cheng, Y. (2013). Studying the 'spiral of silence' status seen from BBS network environment. Jin Tian, 316, 403-405.

Gao, X. C. et al. (2014). From "passive silence" to "active interaction": "double spiral of silence" effect in new media environment. Press, 9, 43-54.

Li, C. S. (2014). The applicability of spiral of silence in the network era. Spread and Copyright, 8, 4-12.

Na, Y. L. (2015). Tweeting the public: journalists' Twitter use, attitudes toward the public's tweets, and the relationship with the public. Information Communication \& Society, 18(4), 443-458. 
Noelle-Neumann. (1977). Turbulences in the Climate of Opinion: Methodological Applications of the spiral of silence theory. Public Opinion Quarterly, 41(2), 143-158.

Sherice, G. et al. (2014). Gay Bullying and Online Opinion Expression: Testing Spiral of Silence in the Social Media Environment. Social Science Computer Review, 32(1), 18-36.

Yang, Z. B. (2014). New 'Silence Spiral' Theory in the Environment of Internet Communication. Journal of Harbin University, 35(7), 95-98.

Zhou, B. H. (2014). Empirical study of college students ' network expression and factors-the 'Spiral of silence' and the 'climate of opinion' as the core. Contemporary Communication, 5, 34-37.

\section{Copyrights}

Copyright for this article is retained by the author(s), with first publication rights granted to the journal.

This is an open-access article distributed under the terms and conditions of the Creative Commons Attribution license (http://creativecommons.org/licenses/by/4.0/). 